\title{
Fluorescence Method for the Detection of Protein Kinase Activity by Using a Zirconium-Based Metal-Organic Framework as an Affinity Probe
}

Jie Bai, ${ }^{*}{ }^{\dagger}, \star$ Liyan Liu, ${ }^{\star}$ Congcong Jia, ${ }^{\dagger}$ Zeping Liu, ${ }^{*}$ Shutao Gao, ${ }^{\dagger} \S$ Yanmei Han, ${ }^{*}$ and Hongyuan Yan ${ }^{*}, \dagger$

$\dagger$ Key Laboratory of Medicinal Chemistry and Molecular Diagnosis, Ministry of Education, Chemistry and Environmental Science, Hebei University, Baoding 071002 , China

$\$$ Medical Comprehensive Experimental Center, College of Public Health, Hebei University, Baoding 071002, China

${ }^{\S}$ Hebei Key Laboratory of Bioinorganic Chemistry, College of Sciences, Agricultural University of Hebei, Baoding 071001, China

*E-mail: bj0427@126.com.

*E-mail: yanhy@hbu.edu.cn. 
Table of Contents:

Apparatus and Reagents

Figure S1 Effect of ACN and the peptide/ATP ratio for the fluorescence intensity 
Apparatus and Reagents

The fluorescence signals were recorded on a spectrophotofluorometer RF-5301PC (Shimadzu, Japan). Cold field emission scanning electron microscopy (SEM) were conducted on JSM-7500F (JEOL Ltd., Japan) at an accelerating voltage of $10.0 \mathrm{kV}$. Powder X-ray diffraction (PXRD) pattern was recorded on a D8 ADVANCE X-ray diffractometer (Bruker, Germany) using $\mathrm{Cu}-\mathrm{K} \alpha \mathrm{X}$-rays at a scanning rate of $3^{\circ} / \mathrm{min}$ in the 2-theta range of $5^{\circ}-50^{\circ}$. The tube voltage and current applied were $40 \mathrm{kV}$ and 40 $\mathrm{mA}$, respectively.

ATP and protein kinase A (PKA, catalytic subunit) were purchased from New England Biolabs(Beverly, MA, USA). GL Biochem Ltd. (Shanghai, China) synthesized PKA-specific substrate peptide1 (TAMRA-LRRASLG, TAMRA-peptide1) and AKT1-specific substrate peptide (TAMRA-CKRPRAASFAE, TAMRA-peptide2). Methanol (MeOH), dimethylformamide (DMF), forskolin, 3-isobutyl-1-methylxanthine (IBMX), and v-akt murine thymoma viral oncogene homolog 1 (AKT1) were purchased from Sigma-Aldrich(St Louis, MO, USA). PKA inhibitor H-89 was supplied by EMD Bioscience(San Diego, CA, USA). Protein extraction kit (C500022) and the Modified Lowry Protein Assay Kit(C504041) were purchased from Sangon Biotech (Shanghai, China). $\mathrm{ZrCl}_{4}$, terephthalic Acid was purchased from Shanghai Aladdin Biochemical Technology Co., Ltd. (Shanghai, China). Protein Kinase (PK) buffer: $50 \mathrm{mmol} \cdot \mathrm{L}^{-1}$ Tris- $\mathrm{HCl}\left(\mathrm{pH} \mathrm{7.5,} 25{ }^{\circ} \mathrm{C}\right), 10$ $\mathrm{mmol} \cdot \mathrm{L}^{-1} \mathrm{MgCl}_{2}, 0.1 \mathrm{mmol} \cdot \mathrm{L}^{-1} \mathrm{EDTA}, 2 \mathrm{mmol} \cdot \mathrm{L}^{-1} \mathrm{DTT}$, and $0.01 \%$ Brij 35. PKA 
storage buffer: $20 \mathrm{mmol} \cdot \mathrm{L}^{-1}$ Tris- $\mathrm{HCl}\left(\mathrm{pH} 7.5,25^{\circ} \mathrm{C}\right), 50 \mathrm{mmol} \cdot \mathrm{L}^{-1} \mathrm{NaCl}, 2 \mathrm{mmol} \cdot \mathrm{L}^{-1}$ DTT, $1 \mathrm{mmol} \cdot \mathrm{L}^{-1} \mathrm{Na}_{2} \mathrm{EDTA}$, and $50 \%$ Glycerol, storage at $-20{ }^{\circ} \mathrm{C}$. AKT1 storage buffer: $50 \mathrm{mmol} \cdot \mathrm{L}^{-1}$ Tris- $\mathrm{HCl}, \mathrm{pH} 7.5,150 \mathrm{mmol} \cdot \mathrm{L}^{-1} \mathrm{NaCl}, 10 \mathrm{mmol} \cdot \mathrm{L}^{-1}$ glutathione, $0.1 \mathrm{mmol} \cdot \mathrm{L}^{-1}$ EDTA, $0.25 \mathrm{mmol} \cdot \mathrm{L}^{-1} \mathrm{DTT}, 0.1 \mathrm{mmol} \cdot \mathrm{L}^{-1} \mathrm{PMSF}$, and $25 \%$ glycerol. Cell lysis buffer: protease inhibitor, dithiothreitol (DTT), and phenylmethylsulfonyl fluoride (PMSF). All other reagents, unless otherwise specified, were of analytical grade and used without further purification. The water used in all experiments was an ultrapure deionized grade $(18.2 \mathrm{M} \Omega \mathrm{cm})$ (produced by the Millipore Milli-Q Integral 5 Water Purification System).
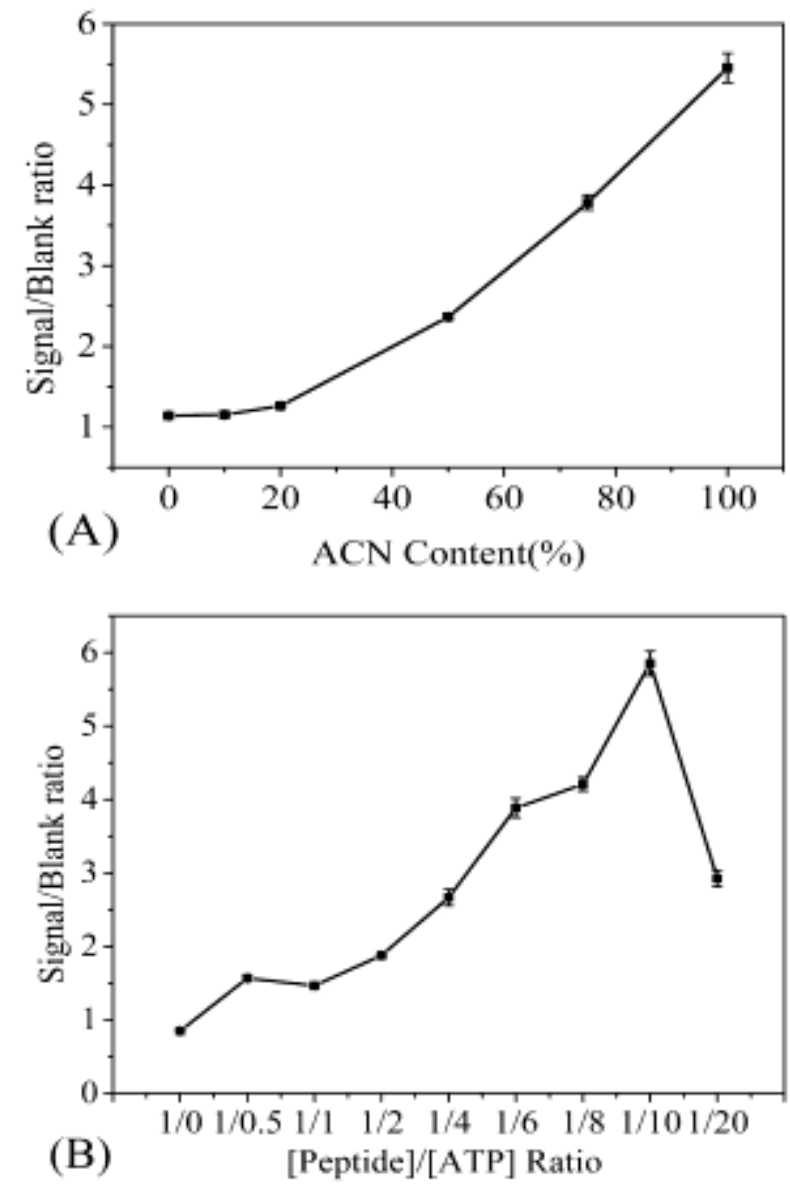

Figure S1 Effect of ACN and the peptide/ATP ratio for the fluorescence intensity 
(A) Effect of ACN; (B) Effect of the peptide/ATP ratio.

The error bars represent the standard deviation of three measurements. 